\title{
Utility of Home-Made Videos in an Adult Epilepsy Clinic
}

Joaquín Ojeda*, Gerardo Gutierrez, Rafael Del Rio Villegas, Vicente Ivañez and Susana López Gallardo

Department of Adult Neurology, Hospital Universitario Infanta Sofia, Madrid, Spain

${ }^{*}$ Corresponding author: Joaquín Ojeda, Epilepsy Monographic Consult, Department of Adult Neurology, Hospital Universitario Infanta Sofia, Madrid, Spain, Tel: +34 9119140 00; E-mail: ojedamd@gmail.com

Rec date: Oct 03, 2016; Acc date: Oct 26, 2016; Pub date: Oct 28, 2016

Copyright: $\odot 2016$ Ojeda J, et al. This is an open-access article distributed under the terms of the Creative Commons Attribution License, which permits unrestricted use, distribution, and reproduction in any medium, provided the original author and source are credited.

\begin{abstract}
Introduction: According to the ILAE, the definition of epilepsy requires the occurrence at least one epileptic seizure. Up to $20 \%$ of patients diagnosed of epilepsy are not actually epileptic. An accurate diagnosis is necessary for a satisfactory management of patients with Epilepsy. New broad access image technologies allow patients and families to record homemade videos helping neurologists in diagnosis.
\end{abstract}

Method: During a two-year period in an epilepsy clinic, consecutive patients were encouraged to record their events with any available device. Instructions for good quality videos were given. Three neurologists/epileptologists watched the videos in clinical session, rated the quality of the recordings following some parameters and made a clinical diagnosis. In a second phase, previous diagnosis was revised.

Results: 314 consecutive patients (relatives) were encouraged to record events. $52 \%$ male. Average age: 46 years. 267 patients had video recording devices available (87\%) (Photo camera: 100\%, cell-phone: 100\%, webcam: $10 \%$, video camera: $30 \%)$. From this group, $135(50 \%)$ felt unable to record events. Reasons given: Low seizure frequency: $60 \%$, seizures short duration: $80 \%$. 50 events from 22 patients recorded. Mean age: 35 years. Seizure frequency three months prior to video deposit was 3, 5 seizures/patient/month. Previous epileptic syndrome diagnosed (based on description/neuroimaging/EEG): 15 focal temporal lobe epilepsy, 4 focal frontal probably symptomatic epilepsy, 3 epileptic encephalopathy.

Type of seizures recorded: Focal motor with typical automatisms: 14 seizures/11 patients. Focal motor with hyperkinetic automatisms: 9 seizures/2 patients. Asymmetrical tonic motor seizures: 4 seizures/1 patient. Focal clonic seizure: 5 seizures/1 patients. Atypical absence seizure: 6 seizures/3 patients. Non-epileptic Seizure (NES): 13 seizures/3 patients. Postural tremor: 1 patient. There was agreement in diagnosis but in one. 18 patients were confirmed in their Diagnosis: Epilepsy misdiagnosis: 4. Three NES and one undetermined tremor.

Conclusion: Homemade videos may be of diagnostic value in epilepsy management. Training in performing good-quality videos is necessary. Webcam long term recordings should be recommended as the best recording option.

Keywords: Seizure; Epilepsy; Homemade; Epilepsy diagnosis; Semiology; Non-epileptic seizure

\section{Introduction}

According to the International League against Epilepsy (ILAE), the definition of epilepsy requires the occurrence of at least one epileptic seizure. A seizure is defined as 'a transient occurrence of signs and/or symptoms due to abnormal excessive or synchronous neuronal activity in the brain' [1]. Diagnosis largely relies in patient's or witnesses' description of signs and symptoms. The clinical diagnosis of epilepsy is not always easy, and is often made after several seizures. These descriptions are often inaccurate or incomplete and may mislead the clinician [2].

Published data confirms that up to $20 \%$ of patients diagnosed of epilepsy are not actually epileptic [3], that is, patients diagnosed of epilepsy have never had seizures, but other type of events. An accurate diagnosis is necessary for a satisfactory management of epileptic and non-epileptic patients.
New broad access image technologies allow patients and families to record homemade videos of their own or their relative's seizures and can help the neurologist in the diagnosis of epilepsy [4], treatment choice and other clinical decisions that will directly affect epilepsy management and patient's and family's quality of life [5].

It seems especially interesting in developing countries, where access to video-monitoring units is not always available or affordable by general population [6].

Concerns regarding privacy may be an obstacle in asking patients or caregivers to record events, especially in those violent and breathtaking situations. Insisting in the importance of an accurate diagnosis, which could be obtained through this useful tool, should help patients finally decide to record. Data protection and personal privacy should be highly guaranteed by the institution, following local and countries legislations, based on respect of patients' principle of nonmaleficence and autonomy. 
Page 2 of 5

\section{Methods}

The primary goal of the study was to assess the feasibility of asking patients with epilepsy (PWE) and/or caregivers to record home videos during the events. The secondary goal was to assess the utility of these recordings in epilepsy diagnosis confirmation, focussing in several items that would determine the quality of video recordings.

We designed a prospective observational study during a two-year period in an adult epilepsy outpatient clinic (2005 to 2006) of the
Spanish National Health Service (Seguridad Social). Consecutive patients with drug resistant epilepsy with defined diagnosis (established at least two years before) as well as relatives and caregivers, were encouraged to record their seizures or clinical events with any available device. Instructions for good-quality videos were given (Table 1). The recordings were transferred to the patient clinical record in following scheduled visits (2-3 months).

\begin{tabular}{|l|}
\hline -Turn on the lights of the room if necessary \\
\hline -Try to record in a steady way, in order to avoid excessive image shaking \\
\hline -During the fit, to check the level of consciousness, ask the patient the following questions: \\
\hline What's your name? \\
\hline What is this I show you? (while a watch, a pen, glasses or a ring is being shown) \\
\hline Remember the words: Bird and football \\
\hline Point the window. Point the door \\
\hline Count up to ten \\
\hline *If possible, try to record several events focusing: The whole body, the patient's face, upper limbs \\
\hline
\end{tabular}

Table 1: Recommendations for good quality video recordings.

In a second phase, three neurologists/epileptologists (JO, VI, GG) from three different hospitals at the moment of the evaluation watched the videos in clinical session, rated the quality of the recordings following fixed parameters (face or whole body recorded, environment illumination, single or multiple fits recorded, early stage of the event recorded) (Table 2) and made a clinical diagnosis. Seizures where classified according to the proposed ILAE seizure organization [1].

Authors compared previous diagnosis obtained from historical clinical records with the clinical and semiological diagnosis obtained after watching homemade video recordings.

\begin{tabular}{|l|l|}
\hline POINTS & (Minimum: 3 points; Max: 8 points) \\
\hline Face recorded & 1 \\
\hline Whole body recorded & 1 \\
\hline Optimum illumination & 1 \\
\hline Single event recorded & 1 \\
\hline Two or more events recorded & 2 \\
\hline Beginning of the event recorded & 2 \\
\hline Post-event recorded & 1 \\
\hline
\end{tabular}

Table 2: Video quality rating.

Patients were asked for specific informed consent for viewing and storing their recordings in the clinic database.

\section{Results}

A total of 314 consecutive patients (or caregivers) were encouraged to record events. $52 \%$ were males. The mean age was $46+/-17$ years.

We found that 267 patients had video recording devices available (87\%) (100\% photo-camera, $100 \%$ cell phone, $10 \%$ webcam, $30 \%$ video-camera). From this group, 135 (50\%) felt unable to record events since the moment of proposal. Several reasons were given: Low seizure frequency $60 \%$, seizures short duration $80 \%$, and caregiver' lack of time spent with patient: $30 \%$. 132 patients accepted the proposition, but finally 110 failed in taking in to the epilepsy clinic any recording. Reasons adduced were (in descending order) not witnessing any seizure, short duration of fits, nocturnal events, emotional blackout.

A total of 50 events from 22 patients were recorded (Table 3). 13 were females. The mean age was $35+/-4$ years. Seizure frequency three months prior to video deposit was 3,5 seizures/patient/month, reflecting a high degree of drug resistant epilepsy of this group of patients. 5 events from 2 patients were discarded and not included in further analysis, due to lack of evidence of clinical event or extreme bad quality of video recording.

All the events recorded were self-limited, and confirmed by relatives/caregivers as similar to usual events.

\section{Quality of video (QOV) recordings}

Regarding to the QOV, the mean rating was 4,6 points in a scale of 8 to 3 points (Table 2) (8, maximum quality qualification). $9 \%$ had excellent QOV (rating 8-7), 64\% had medium QOV (rating 6-5), and $27 \%$ had low QOV (4-3). The best video recordings corresponded to both patients who used a webcam, playing long-term recordings at workplace and at home respectively. Webcam recordings obtained 
Page 3 of 5

better QOV ratings, compared to the other types of video devices, but no statistic significant association was found.

Types of seizures recorded were classified as follows: Focal seizures with consciousness impairment with oral and manual automatisms: 12 seizures, 10 patients. Focal seizures without consciousness impairment and oral automatisms: 2 seizures, 1 patient. Focal motor with hyper motor automatisms: 9 seizures, 2 patients. With asymmetrical tonic motor seizures: 4 seizures, 1 patient. Focal motor clonic seizures: 5 seizures, 1 patient. Atypical absence seizure: 6 seizures, 3 patients. Non-epileptic seizure (NES): 13 seizures, 3 patients. There was a difficult to defined event, mimicking a postural tremor in one patient. Only one patient was recorded with a focal seizure evolving to bilateral convulsions.

Previous epilepsy syndromes diagnosed to patients recorded (based on patients'-relatives' description, neuroimaging and EEG recordings) were as follows: 15 focal temporal lobe epilepsy (9 medial temporal lobe epilepsy with hippocampal sclerosis, 5 cryptogenic, 1 posttraumatic), 4 focal frontal cryptogenic epilepsy, 3 epileptic encephalopathy. There were no suspected NES in these patients.

\begin{tabular}{|c|c|c|c|c|c|c|c|}
\hline Patient & $\begin{array}{l}\text { Recording } \\
\text { device }\end{array}$ & $\begin{array}{l}\text { Seine Frequency } \\
\text { (monthly) }\end{array}$ & Total recordings & Video Quality & $\begin{array}{l}\text { Best video } \\
\text { Qualification }\end{array}$ & $\begin{array}{l}\text { Pre-post } \\
\text { concordance }\end{array}$ & Seizure Type \\
\hline \#1 & Wcam, cell & 7 & 7 & 6 & 8 & YES & FSHA \\
\hline \#2 & pho & 3 & 2 & 6 & 6 & YES & FSTA \\
\hline \#3 & pho & 1 & 5 & 6 & 7 & NO & NES \\
\hline \#4 & Pho & 2 & 4 & 5 & 5 & YES & FSATM \\
\hline \#5 & Vidcam & 0,5 & 2 & 4 & 5 & YES & FCS \\
\hline \#6 & Pho & 10 & 4 & 8 & 8 & NO & NES \\
\hline \#7 & Pho & 0,1 & 1 & 2 & 2 & YES & FSTA \\
\hline \#8 & Pho & 3 & 1 & 2 & 2 & YES & AM \\
\hline \#9 & Pho & 1 & 1 & 5 & 5 & NO & FSTA \\
\hline$\# 10$ & Pho & 0,8 & 4 & 7 & 8 & YES & AM \\
\hline \#11 & Pho & 0,5 & 2 & 7 & 8 & YES & FSHA \\
\hline \#12 & Pho & 1 & 1 & 4 & 3 & YES & FSTA \\
\hline \#13 & Pho & 0,3 & 1 & 4 & 4 & YES & FSTA \\
\hline \#14 & Pho & 0,5 & 1 & 3 & 3 & YES & FSTA \\
\hline \#15 & Wcam & 5 & 6 & 6 & 3 & YES & FSTA \\
\hline \#16 & Pho & 30 & 3 & 3 & 3 & NO & NES \\
\hline \#17 & V cam & 3 & 1 & 2 & 2 & Undetermined & Tremor \\
\hline \#18 & Pho & 0,4 & 1 & 4 & 4 & YES & FSTA \\
\hline \#19 & Pho & 2 & 1 & 3 & 3 & YES & FSTA \\
\hline \#20 & Pho & 6 & 1 & 7 & 8 & YES & AM \\
\hline \#21 & Pho & 1 & 1 & 6 & 7 & YES & FSTA \\
\hline \#22 & Pho & 1 & 1 & 4 & 4 & YES & FSTA \\
\hline
\end{tabular}

Table 3: List of patients: Description.

In the second phase of the study, and after thoroughly evaluation by the research team, there was agreement among neurologists in the clinical and semiological diagnosis of all home recorded events but in one (patient with tremor: Action tremor vs. focal clonic seizure in a patient with epileptic encephalopathy).
All the three patients newly diagnosed with NES were female and had a previous diagnosis of cryptogenic temporal lobe epilepsy. Seizure frequency rate three months prior to video deposit in these three patients was 13, 6 seizures/patient/month . A total of 13 events were recorded. Mean QOV rate of 6 points (medium). 
NES were more likely to be recorded and yield better QOV, compared to patients who suffered epileptic seizures. We found no statistical association, likely due to scarce number of patients.

18 patients were confirmed in their diagnosis after viewing homemade recordings in clinical session. Three patients were defined as misdiagnosed; all of them presented non-epileptic seizures, and caregivers confirmed those events as usual ones. One was considered with no defined diagnosis (no agreement in the nature of the event among experts).

\section{Discussion}

Epilepsy remains a clinical diagnosis. A correct diagnosis is essential for an adequate management of PWE. Neurologists rely on patient's or witnesses' description of seizures to make a correct diagnosis. Unfortunately, these descriptions are often inaccurate or incomplete, even when made by medical or paramedical staff $[3,7]$ and may mislead the clinician. Some patients have not undergone an epileptic seizure but other type of event. A correct diagnosis can spare the patient unnecessary tests or treatments [8]. Solid diagnosis of epilepsy is considered only on expert video-EEG monitoring basis [9-11]. It is known, however, that video-EEG has several disadvantages: Only two thirds of the patients referred seize during the monitoring, despite they are usually drug-resistant patients; video-EEG is not always available in primary or secondary centers [10-12], as well as not affordable for many patients and their families [6]. Video-EEG based diagnosis has not yet proved a perfect inter-rater reliability in distinguishing epileptic seizures from NES $[13,14]$. Some studies, looking forward to minimize the bias of local tradition semiology interpretations, found that accuracy of interpretation based on patients'-caregivers descriptions was only $54 \%$. After reviewing video EEG recordings the accuracy increased to $85 \%$.

New video-recording devices are broadly used by general population, and may help neurologist in accurately diagnose epilepsies $[4,15,16]$. mHealth (also written as m-health or mobile health) is a term used for the practice of medical and public health, supported by mobile devices. The term is most commonly used in reference to using mobile communication devices, such as smartphones, for health services and information but it could include the recording of images or videos to help the clinician in diagnosis [17]. In Spain there are more cell-phones that population $[18,19]$. Many of these recording devices are readily available and can record high quality videos, sometimes better than many of the video cameras used in a conventional video-EEG monitoring and surely than those used several years ago [20]. Thus, this technology allows obtaining valuable clinical information of the semiology of the seizures of the patients. Also it allows the clinical to have an insight into the patients' home or workplace and obtain information about how their epilepsy interferes with daily activities.

$87 \%$ of patients had recording devices. Only half of them felt able to record the events (132 patients). From those only 22 patients (17\%) finally shot videos. In a recent study taking place in India, they found a much better rate of home video recording success. Out of a total of 340 patients, 312 (91\%) could provide home videos (624 videos). They were PWE that were waiting for a Video EEG monitoring to be done (for seizure type characterization or on-invasive presurgical evaluation) [4]. In our study, only $17 \%$ of patients recorded home videos, despite their drug resistant status and seizure frequency high rate.
Three patients suffered non-epileptic seizures instead of temporal seizures. In these patients, NES were more likely to be recorded and performed better quality video recording, compared to patients who suffered epileptic seizures. In these three patients the homemade video shifted the diagnosis and clinical management and improved their clinical performance after introducing other therapeutic approaches. This scenario is common in epilepsy centers after video EEG monitoring $[13,21,22]$. Perhaps, as referred in our study, home video recordings could spare to the patient and institutions a number of video EEG studies.

Only two patients of the study used webcams, with long term recordings. Only these two patients provided seizure onset recordings. Recording of seizure onset and several seizure shots, were responsible of the excellent QOV rating in this two patients.

In our sample there were not primary generalized seizures recordings (only one secondarily generalized seizure). This could be explained because most generalized epilepsies are seizure free/drugsensitive and are less referred to epilepsy clinic.

There are several limitations to homemade video diagnosis of epilepsy. While consistent diagnosis of epilepsy is obtained by longterm video-EEG monitoring [9], homemade videos can only register a little number of the patient's events. In the other hand, they result cost-effective and may avoid emotional stress related to admission to a video EEG monitoring unit.

Patients with uncontrolled seizures are more likely to summit recordings to their neurologist. Furthermore, the same patient can have several types of seizures or a combination of epileptic seizures with events of other nature [21,22-24], so it is possible to misdiagnose some patients on the basis of a reduced number of events recorded. As expected, there was a patient which the semiology/nature of the event did not meet agreement among neurologists. This reflects also that homemade videos are not perfect, and that interobserver reliability is also low, even if they are experts [9].

\section{Conclusion}

In conclusion, we think that homemade videos of seizures can be of diagnostic value in epilepsy diagnosis and management. Homemade videos are inexpensive, widespread, and features high definition recordings. It is important to train patients and relatives in performing good-quality videos to optimize this useful tool. Webcam long term recording should be the first choice.

Home video recordings are not intended to replace video-EEG monitoring studies, but probably could avoid undergoing them in some selected cases.

Data protection and personal privacy should be highly guaranteed to the patient by the doctor and the institution, based on respect of patients' principle of nonmaleficence and autonomy [24]. Furthermore, a fluent and trust based doctor patient relationship, should encourage patients to optimized this potentially useful tool.

In addition, we subjectively perceived that families and patients felt more involved in the treatment and patient-physician relationship improved.

Finally, we must remind relatives and caregivers that patient's safety is always first, and of course, comes before a great video recording. 


\section{Disclosure}

Dr. Ojeda has received funding for travel from UCB Pharma, and grants for activities sponsored by UCB Pharma, Boehringer Ingelheim, GSK and Bial.

Dr. Gutierrez Gutierrez has received funding for travel from Eisai.

Dr. Ivanez has received funding for travel from UCB and grants for activities sponsored by UCB Pharma.

Dr. Lopez Gallardo: No disclosures.

Dr. Rafael del Rio: No disclousures.

\section{References}

1. Berg AT, Berkovic SF, Brodie MJ, Buchhalter J, Cross HJ, et al. (2010) Revised terminology and concepts for organization of seizures and epilepsies: report of the ILAE Commission on Classification and Terminology, 2005-2009. Epilepsia 51: 676-685.

2. Mannan JB, Wieshmann UC (2003) How accurate are witness descriptions of epileptic seizures? Seizure 12: 444-447.

3. Scheepers B, Clough P, Pickles C (1998) The misdiagnosis of epilepsy: findings of a population study. Seizure 7: 403-406.

4. Dash D, Sharma A, Yuvraj K, Renjith A, Mehta S, et al. (2016) Can home video facilitate diagnosis of epilepsy type in a developing country? Epilepsy Res 125: 19-23.

5. Johansen JR, Lindahl G, Sandstedt P (1999) Home-video observation of seizures in children with epilepsy-impact on quality of family life. Seizure 8: 356-357.

6. Chemmanam T, Radhakrishnan A, Sarma SP, Radhakrishnan K (2009) A prospective study on the cost-effective utilization of long-term inpatient video-EEG monitoring in a developing country. J Clin Neurophysiol 26: 123-128.

7. Gonsalves B, Paller KA (2002) Mistaken memories: remembering events that never happened. Neuroscientist 8: 391-395.

8. Landau WM, Nelson DA (1996) Clinical neuromythology XV. Feinting science: Neurocardiogenic syncope and collateral vasovagal confusion. Neurology 46: 609-618.

9. Nordli DR Jr (2006) Usefulness of video-EEG monitoring. Epilepsia 47: 26-30.
10. Alsaadi TM, Thieman C, Shatzel A, Farias S (2004) Video-EEG telemetry can be a crucial tool for neurologists experienced in epilepsy when diagnosing seizure disorders. Seizure 13: 32-34.

11. Asano E, Pawlak C, Shah A, Shah J, Luat AF, et al. (2005) The diagnostic value of initial video-EEG monitoring in children-review of 1000 cases. Epilepsy Res 66: 129-135.

12. Benbadis SR, O'Neill E, Tatum WO, Heriaud L (2004) Outcome of prolonged video-EEG monitoring at a typical referral epilepsy center. Epilepsia 45: 1150-1153.

13. Mohan KK, Markand ON, Salanova V (1996) Diagnostic utility of video EEG monitoring in paroxysmal events. Acta Neurol Scand 94: 320-325.

14. Benbadis SR, LaFrance WC Jr, Papandonatos GD, Korabathina K, Lin K, et al. (2009) Interrater reliability of EEG-video monitoring. Neurology 73: 843-846.

15. Parikh R, Wong R (2007) Video phone diagnosis of 'funny turns'. Age Ageing 36: 233-234.

16. Zeiler SR, Kaplan PW (2009) Our digital world: camera phones and the diagnosis of a seizure. Lancet 373: 2136.

17. Vital Wave Consulting (2009) mHealth for Development: The Opportunity of Mobile Technology for Healthcare in the Developing World. United Nations Foundation, Vodafone Foundation 9.

18. Spanish Commission of Telecommunications Market (2009) Annual report.

19. National Institute of Statistics. Spanish Total Population (2010) Official Data at January 1 st.

20. Samuel M, Duncan JS (1994) Use of the hand held video camcorder in the evaluation of seizures. J Neurol Neurosurg Psychiatr 57: 1417-1418.

21. Syed TU, Lafrance WC Jr, Kahriman ES (2011) Can semiology predict psychogenic nonepileptic seizures? a prospective study. Ann Neurol 69: 997-1004.

22. Benbadis SR, Agrawal V, Tatum WO 4th (2001) How many patients with psychogenic nonepileptic seizures also have epilepsy? Neurology 57: 915-917.

23. Beniczky SA, Fogarasi A, Neufeld M, Andersen NB, Wolf P, et al. (2012) Seizure semiology inferred from clinical descriptions and from video recordings. How accurate are they? Epilepsy Behav 24: 213-215.

24. Beauchamp TL, Childress JF (2001) Principles of Biomedical Ethics (5th edn.) New York: Oxford University Press. 